\title{
STELLAR PHOTOMETRY WITH A PERFECT CCD
}

\author{
Bjarne Thomsen and Frank Grundahl Jensen \\ Institute of Physics and Astronomy, Aarhus University
}

We have developed algorithms for the construction of a simulated globular cluster, as it would appear on a frame obtained with a perfect CCD. This has allowed us to compare the absolute errors in the photometry of two different programs, DAOPHOT II (Stetson 1991, 1994) and SPS (Janes and Heasley 1993), with a special emphasis on criteria for selecting stars with the best photometry.

\section{THE SIMULATED GLOBULAR CLUSTER}

We assume (Thomsen and Frandsen 1983) that the shape of the Optical Transfer Function (OTF) can be represented by

$$
\begin{aligned}
& \operatorname{OTF}(u, v)=a \mathrm{H}(\omega) \exp (-2 \pi \theta) \\
& \theta=\alpha u+\beta v \\
& \mathrm{H}(\omega)=\exp \left(-(\omega / \gamma)^{\mathrm{P}}\right) \\
& \omega^{2}=u^{2}+\mathrm{v}^{2}+\mathrm{a}_{2}\left(\mathrm{u}^{2}-\mathrm{v}^{2}\right)+2 \mathrm{~b}_{2} \mathrm{uv}
\end{aligned}
$$

The location of the PSF is determined by $(\alpha, \beta)$ and $\left(a_{2}, b_{2}\right)$ is a measure of the direction and size of the ellipticity of the PSF. The adopted FWHM of the PSF was 3.5 pixels.

The stellar positions in the simulated frame were created as the projection of a Plummer model $\left(R_{c}=100\right.$ pixels $)$ for the spatial density of stars. The stellar magnitudes were obtained from a two-segment luminosity function, with one segment representing the giants and the other the main-sequence stars. The parameters defining the luminosity function were estimated from data in Bergbush and VandenBerg (1992). A total of 200,000 stars, between instrumental magnitudes 10 and 23, were added to the frame. The gain and readout noise were set to 3 $\mathrm{e}^{-/}$ADU and $7.5 \mathrm{e}^{-}$, respectively. Subsequently we added a constant night-sky value, a random Poissonian photon noise, and finally a random Gaussian readout noise.

\section{DISCUSSION}

Our main result from the comparison is that the two programs seem to deliver rather similar results when comparing the bulk of the photometry. Both programs find roughly the same number of stars, and the photometric accuracy is also similar. The faint-end bias reported by Schechter et al. (1993) seems to have been removed by the addition to ALLSTAR of a repeated sky determination, as suggested by Parker (1991).

However, in both cases it is evident that a large fraction of the detected stars has a relatively poor photometric accuracy. Approximately $30 \%$ deviate more than 0.2 mag from the 
true value. In the world of real photometry we do not know the true magnitude of a star, and thus we need some criteria for selecting the fraction that has the best photometric accuracy.

ALLSTAR offers various quality indicators. SIGMA is the estimated standard error of the magnitude. $\mathrm{CHI}$ is the ratio of the observed pixel-to-pixel scatter of the fitting residuals to the expected scatter. SHARP is a measure of how well the observed profile of a given star matches that of the PSF. CHI should be close to one, and SHARP is expected to be close to zero. In addition we have implemented two new shape parameters, $A_{2}$ and $B_{2}$. They measure the ellipticity along the coordinate directions and the diagonal directions, respectively.

Through cuts in (SIGMA, CHI, SHARP, $\mathrm{A}_{2}, \mathrm{~B}_{2}$ )-space we isolated stars with the best photometry. After a few experiments we arrived at the following useful limits on the ALLSTAR parameters: CHI $<1.4,-0.3<$ SHARP $<0.2, \sqrt{ }\left(\mathrm{A}_{2}{ }^{2}+\mathrm{B}_{2}{ }^{2}\right)<0.15$, and SIGMA $<1.1 *$ MEDIAN(SIGMA $(\mathrm{m}))+0.001 .30 \%$ of the total number of stars detected by DAOPHOT satisfies these limits, but $6 \%$ of the selected stars still deviates more than 0.2 mag from the true value. Omitting these, the mean absolute deviation (MAD) of the remaining sample is 0.03 mag. Our final sample contains 2189 stars, primarily the brightest, but it still contains stars more than 2 mag fainter than the cluster turn-off.

SPS does not provide as many useful indicators as ALLSTAR, and thus the possibility of rejecting stars with poor photometry is smaller. SIGMA is again the standard error. ADD is the number of additional stars that is fitted simultaneously with the current star. We adjusted the bounds on SIGMA and ADD until we arrived at the following limits: ADD $<10$ and SIGMA $<0.1 .30 \%$ of the stars detected by SPS satisfies these limits, but now $12 \%$ of the selected stars deviates more than 0.2 mag. Omitting these, as well as stars fainter than 17 mag, the MAD of the remaining sample is $0.04 \mathrm{mag}$. For the faint-end stars the two SPS constraints are less efficient in rejecting stars with poor photometry than are the four ALLSTAR constraints. The opposite trend seems to apply for the bright-end stars.

After the selection process most of the remaining stars with large deviations are found to be too bright by both programs. This was actually our main motivation for implementing $A_{2}$ and $\mathrm{B}_{2}$ in ALLSTAR, and they did in fact help to remove many stars with large deviations. The number of deviating stars are at least equally abundant in the SPS photometry. Even though we have not been able to identify the cause of the large deviations, it is still important to find a way to locate these stars accurately, since they might contribute considerably to the scatter in an observed color-magnitude diagram, especially near the turn-off region.

\section{REFERENCES}

Bergbush, P. and VandenBerg, D. A. 1992 ApJS 81, 163

Janes, K. A. and Heasley, J. N. 1993 PASP 105, 527

Parker, J. Wm. 1991 PASP 103, 243

Schechter, P. L., Mateo, M. and Saha, A. 1993 PASP 105, 1342

Stetson, P. B. 1991 in Proceedings of the 3rd ESO/ST-ECF Data Analysis Workshop, P. J.

Grosbol and R. H. Warmels, eds., ESO, Garching, p. 187

Stetson, P. B. 1994 PASP 106, 250

Thomsen, B. and Frandsen, S. 1983 AJ 88, 789 